\title{
AN INEQUALITY FOR THE SPECTRAL RADIUS OF MARKOV PROCESSES
}

\author{
By SADAO SATO
}

\section{Introduction.}

Let $A$ be a second-order uniformly elliptic operator in a bounded domain $D$. Consider the eigenvalue problem

$$
A u+\lambda u=0
$$

with mixed boundary conditions:

$$
\begin{aligned}
u=0 & \text { on } \Gamma_{1} \\
\frac{\partial u}{\partial n}+\alpha(x) u=0 & \text { on } \Gamma_{2},
\end{aligned}
$$

where $n$ stands for the outer normal and $\partial D=\Gamma_{1} \cup \Gamma_{2}$. Let $\lambda_{0}$ be the first eigenvalue. When $A$ is symmetric, J. Barta proved that

$$
\inf \{-A u / u\} \leqq \lambda_{0} \leqq \sup \{-A u / u\},
$$

where $u$ is any positive $C^{2}$-function satisfying the same boundary conditions (1.2) (see [1]).

When $A$ is nonsymmetric, M.H. Protter and H.F. Weinberger [7] proved the left hand of (1.3) for any function $u$ satisfying

$$
\begin{aligned}
u>0 & \text { on } \quad D \cup \partial D \\
\frac{\partial u}{\partial n}+\alpha(x) u \geqq 0 & \text { on } \Gamma_{2} .
\end{aligned}
$$

Let $\alpha(x)$ be positive. Then there exists a diffusion process with the generator $A$ whose domain is the collection of $C^{2}$-functions satisfying (1.2).

For a Markov process, we can define the spectral radius $\lambda_{0}$ by

$$
\lambda_{0}=\lim _{t \rightarrow \infty}-\frac{1}{t} \log \left\|T_{t}\right\|
$$

where $\left\{T_{t}\right\}$ is the associated semigroup and $\left\|T_{t}\right\|=\sup _{x} T_{t} 1(x)$.

Our main purpose is to prove the inequality (1.3) for the spectral radius of a Markov process satisfying some conditions. We will show that the spectral

Recelved January 31, 1984 
radius is equal to the first eigenvalue if the first eigenfunction exists. Thus as a corollary we can see that the inequality (1.3) holds for a nonsymmetric diffusion process. For the proof, the existence of a stationary measure will play a fundamental role.

\section{Notations.}

Let $\left(P_{x}, X_{t}\right)$ be a right continuous strong Markov process on a state space $S$ which is a locally compact separable Hausdorff space. Then the resolvent operator $G_{\alpha}$ of $\left(X_{t}\right)$ is defined by

$$
G_{\alpha} u(x)=E_{x}\left[\int_{0}^{\sigma} e^{-\alpha s} u\left(X_{s}\right) d s\right],
$$

where $u$ is a bounded measurable function, and $\sigma$ is the life time of $\left(X_{t}\right)$. Let $\bar{S}$ be the one point compactification of $S$, and denote

$$
\bar{S}=S \cup\{\partial\} \text {. }
$$

In the probabilistic sense, $\partial$ is called the death point and related to the life time $\sigma$ by

$$
X_{t} \in S \text { for all } t<\sigma \text { and } X_{t}=\partial \text { for all } t \geqq \sigma .
$$

We define the spaces of real-valued functions with the supremum norm as follows :

$$
\begin{aligned}
& C(S)=\{u ; u \text { is bounded continuous on } S\}, \\
& C_{+}(S)=\{u \in C(S) ; u \geqq 0 \text { and } u(x)>0 \text { for some } x \in S\}, \\
& B(S)=\{u ; u \text { is bounded Borel measurable on } S\}, \\
& B_{+}(S)=\{u \in B(S) ; u \geqq 0 \text { and } u(x)>0 \text { for some } x \in S\} .
\end{aligned}
$$

We also define the spaces of measures on the topological Borel field as follows :

$$
\begin{aligned}
& M(\bar{S})=\{m ; m \text { is a bounded Borel measure on } \bar{S}\}, \\
& \Pi(\bar{S})=\{P ; P \text { is a probability measure on } \bar{S}\} .
\end{aligned}
$$

In the most of the paper we assume the following conditions.

(A. 1) $\quad\left(X_{t}\right)$ is a Feller process, that is $G_{\alpha}: C(S) \rightarrow C(S)$.

(A.2) $\quad \lim _{x \rightarrow \partial} G_{\alpha} 1(x)=0$ (if $S$ is non-compact).

If $S$ is compact, we demand $P_{x}(\sigma<\infty)>0$ for some $x \in S$.

(A.3) For every non-void open set $G$ in $S$ and $x \in S, P_{x}\left(\sigma_{G}<\infty\right)>0$,

where $\sigma_{G}$ is the first hitting time for $G$. 
We set $G_{\alpha} u(\partial)=0$ for every $u \in B(S)$. Under the conditions (A.1) and (A.2), we can regard $G_{\alpha}$ as the operator on $C(\bar{S})$. We denote by $G_{\alpha}^{*}$ the dual operator of $G_{\alpha}$ on $M(\bar{S})$. Note that the condition (A.2) implies that

$$
G_{\alpha}^{*} m(\partial)=0 \text { for every } m \in M(\bar{S}) .
$$

and the condition (A.3) implies that

$$
\begin{aligned}
& G_{\alpha} u>0 \text { for every } u \in C_{+}(\bar{S}) \\
& \text { (support } \left.\left(G_{\alpha}^{*} m\right)=S \text { for every } m \in M(S)\right) .
\end{aligned}
$$

LEMMA 2.1. For every $u \in B(S)$, we have

$$
G_{\alpha}^{n} u(x)=E_{x}\left[\int_{0}^{\sigma} e^{-\alpha s} s^{n-1} u\left(X_{s}\right) d s\right] /(n-1) ! .
$$

Proof. Though this formula is well-known, we give a proof for the convenience. Since $\left\|G_{\alpha} u\right\| \leqq\|u\| / \alpha$, we can define

$$
v=\sum_{n=1}^{\infty} \lambda^{n} G_{\alpha}^{n} u \quad \text { for } \quad|\lambda|<\alpha .
$$

By the resolvent equation, we can easily see

Therefore we have

$$
v=\lambda G_{\alpha-\lambda} u \text {. }
$$

$$
\begin{aligned}
\sum_{n=1}^{\infty} \lambda^{n} G_{\alpha}^{n} u & =\lambda E_{x}\left[\int_{0}^{\sigma} e^{-\alpha s+\lambda s} u\left(X_{s}\right) d s\right] \\
& =\sum_{n=1}^{\infty} \lambda^{n} E_{x}\left[\int_{0}^{\sigma} e^{-\alpha s} s^{n-1} u\left(X_{s}\right) d s\right] /(n-1) !
\end{aligned}
$$

\section{Spectral radius and Barta's inequality.}

At the first we consider the semigroup $T_{t}$ and the resolvent $G_{\alpha}$ as the operators on $B(S)$.

Since $\left\|T_{t}\right\|=\sup _{x \in S}\left\{P_{x}(t<\sigma)\right\}$ is submultiplicative in $t$, there exists the limit

$$
\lambda_{0}=\lim _{t \rightarrow \infty}-\frac{1}{t} \log \left\|T_{t}\right\|
$$

which will be called the spectral radius of the Markov process $\left(X_{t}\right)$.

THEOREM 3.1.

$$
\begin{aligned}
\lambda_{0} & =\lim _{n \rightarrow \infty}\left\|G_{\alpha}^{n}\right\|^{-1 / n}-\alpha \\
& =\sup \left\{\lambda ; \sup _{x \in S} E_{x}\left[e^{\lambda \sigma}\right]<\infty\right\} .
\end{aligned}
$$


Proof. We denote the right hands of (3.2) by $\lambda_{G}$ and $\lambda_{F}$ respectively. Note that $\lim _{n \rightarrow \infty}\left\|G_{\alpha}^{n}\right\|^{-1 / n}$ is the spectral radius of $G_{\alpha}$. Therefore $T_{\lambda}=\sum_{n=1}^{\infty}(\lambda+\alpha)^{n-1} G_{\alpha}^{n}$ is a continuous operator on $B(S)$ for any $\lambda<\lambda_{G}$. From Lemma 2.1, the norm is given by

$$
\left\|T_{\lambda}\right\|=\sup _{x \in S} T_{\lambda} 1(x)=\sup _{x \in S}\left\{E_{x}\left[e^{\lambda \sigma}\right]-1\right\} / \lambda .
$$

Thus we have $\lambda_{G} \leqq \lambda_{F}$.

If $\lambda<\lambda_{F}$, we have

$$
e^{\lambda t}\left\|T_{t}\right\|=e^{\lambda t} \sup _{x} P_{x}(t<\sigma) \leqq \sup _{x} E_{x}\left[e^{\lambda \sigma}\right]<\infty .
$$

This implies $\lambda \leqq \lambda_{0}$ and so $\lambda_{F} \leqq \lambda_{0}$.

If $\lambda<\lambda_{0}$, we have $\left\|T_{t}\right\| \leqq \exp (-\lambda t)$ for large $t$. Since

$$
\left\|G_{\alpha}^{n}\right\| \leqq \int_{0}^{\infty} e^{-\alpha t} t^{n-1}\left\|T_{t}\right\| d t /(n-1) !,
$$

we can easily obtain $\lambda \leqq \lambda_{G}$. Thus the theorem is proved.

COROLLARY 3.2. The following conditions are equivalent:

(i) $\lambda_{0}>0$,

(ii) $\left\|T_{t}\right\|<1$ for some $t>0$,

(iii) $\left\|G_{\alpha}\right\|<1 / \alpha$ for some $\alpha>0$,

(iv) $\sup _{x} E_{x}[\sigma]<\infty$.

Remark 1. The expression $\lambda_{F}$ is due to A. Friedman. He proved that $\lambda_{F}$ is the principal eigenvalue, when $\left(X_{t}\right)$ is a smooth diffusion process and $S$ is a bounded domain in $R^{n}$ with $C^{2}$-boundary (see [3]). Note that the equality (3.2) does not hold for a semigroup on $C(S)$ in general.

THEOREM 3.3. For any $u \in B_{+}(S)$, we have

$$
\lambda_{0} \leqq \sup \left\{u / G_{\alpha} u\right\}-\alpha .
$$

Suppose that $u$ is uniformly positive on $S$. Then we have

$$
\inf \left\{u / G_{\alpha} u\right\}-\alpha \leqq \lambda_{0} .
$$

Proof. Set $\lambda=\sup \left\{u / G_{\alpha} u\right\}$. Then we have $u \leqq \lambda^{n} G_{\alpha}^{n} u$. Thus for some $x \in S$, we have

$$
0<u(x)^{1 / n} \leqq \lambda\left\|G_{\alpha}^{n}\right\|^{1 / n}\|u\|^{1 / n},
$$

which proves (3.3). Set $\lambda=\inf \left\{u / G_{\alpha} u\right\}$. If $\lambda=0$, then (3.4) is trivial. If $\lambda>0$, then we have

$$
0<(\inf u) \cdot \lambda^{n} G_{\alpha}^{n} 1 \leqq \lambda^{n} G_{\alpha}^{n} u \leqq u
$$

Therefore we obtain

$$
0<(\inf u)^{1 / n} \cdot \lambda\left\|G_{\alpha}^{n}\right\|^{1 / n} \leqq\|u\|^{1 / n},
$$


which proves (3.4).

Remark 2. By Theorem 3.3, we have shown that the right hand side of the Barta's inequality (1.3) holds for every Markov process. In particular (3.4) implies

$$
1 / \sup _{x} E_{x}[\sigma] \leqq \lambda_{0}
$$

However, for the proof of (3.4) for every positive function $u$, we need the conditions (A.1)-(A.3) for the Markov process.

LEMMA 3.4. Let the conditions (A.1) and (A.3) be satisfied. In order that $\lambda_{0}$ be positive, it is necessary and sufficient that

$$
\begin{aligned}
& \lim _{x \rightarrow \partial} \sup _{\alpha} 1(x)<1 / \alpha \\
& \text { (or } P_{x}(\sigma<\infty)>0 \text { for some } x \in S \text { of } S \text { is compact) }
\end{aligned}
$$

Proof. From Corollary 3.2 the necessity is obvious. For the sufficiency, we must prove $\sup G_{\alpha} 1<1 / \alpha$. Suppose that $\left\|G_{\alpha}\right\|=1 / \alpha$. Since $G_{\alpha} 1$ is continuous, there exists a point $y \in S$ such that $G_{\alpha} 1(y)=1 / \alpha$ by (3.5). Let $k=$ $\left(\lim \sup _{x \rightarrow \partial} G_{\alpha} 1(x)+\alpha^{-1}\right) / 2$ and $G=\left\{x ; G_{\alpha} 1(x)<k\right\}$. By the strong Markov property, we have

$$
\alpha^{-1}=G_{\alpha} 1(y) \leqq \alpha^{-1} P_{y}\left(\sigma_{G}=\infty\right)+k P_{y}\left(\sigma_{G}<\infty\right),
$$

which contradicts to the assumption (A.3). If $S$ is compact, the above condition implies that $G=\left\{x ; G_{\alpha} 1(x)<\alpha^{-1}-\varepsilon\right\}$ is a nonvoid open set for some $\varepsilon>0$. If $\left\|G_{\alpha}\right\|=\alpha^{-1}$, then we have for some $y$

$$
\alpha^{-1}=G_{\alpha} 1(y) \leqq \alpha^{-1} P_{y}\left(\sigma_{G}=\infty\right)+\left(\alpha^{-1}-\varepsilon\right) P_{y}\left(\sigma_{G}<\infty\right),
$$

which completes the proof.

LEMMA 3.5. If $\lambda_{0}$ is positive, then we have

$$
\sup _{x} E_{x}\left[\exp \left(\lambda_{0} \sigma\right)\right]=+\infty .
$$

Under the conditions (A.1) and (A.3), we have

$$
\lambda_{0}<+\infty \text {. }
$$

Proof. Define

$$
T_{\lambda}=\int_{0}^{\infty} d t \exp (\lambda t) T_{t}
$$

Then we have

$$
\left\|T_{\lambda}\right\|=\left(\sup _{x} E_{x}\left(e^{\lambda \sigma}\right)-1\right) / \lambda .
$$

Suppose that $\sup E_{x}\left[\exp \left(\lambda_{0} \sigma\right)\right]$ be finite. Then $T_{\lambda_{0}}$ is a bounded operator. Since 
$T_{\lambda_{0}+\varepsilon}=\sum_{n=1}^{\infty} \varepsilon^{n-1} T_{\lambda_{0}}^{n}, T_{\lambda_{0}+\varepsilon}$ is bounded for $0<\varepsilon<1 /\left\|T_{\lambda_{0}}\right\|$. However this means $\sup E_{x}\left[\exp \left(\left(\lambda_{0}+\varepsilon\right) \sigma\right)\right]<+\infty$, which is a contradiction. Let (A.1) and (A.3) be satisfied. Let $u$ be a continuous function with compact support. From (2.6) and Theorem 3.3, we obtain (3.7).

By Lemma 3.4 and 3.5 we know that $\lambda_{0}$ is a finite positive number under the conditions (A.1)-(A.3). Then the Green operator $G=G_{0}$ is continuous operator on $B(S)$ (or $C(\bar{S})$ ). In the following, we use $G$ instead of $G_{\alpha}$.

Theorem 3.6. Assume that the conditions (A.1)-(A.3) be satisfied. Then there exists a probability measure $P$ on $S$ such that

$$
P=\lambda_{0} G^{*} P,
$$

where $G^{*}$ is the dual operator of $G$.

Proof. For $m \in M(\bar{S})$, we define

$$
K_{\lambda} m=\sum_{n=0}^{\infty} \lambda^{n} G^{* n} m \text {. }
$$

If $\lambda<\lambda_{0}$, we have

$$
K_{\lambda} m=m+\lambda G^{*} K_{\lambda} m
$$

and

$$
K_{\lambda} m(\bar{S})=\int E_{x}\left[e^{\lambda \sigma}\right] d m(x) .
$$

From Lemma 3.5, we can take the sequences $\left\{x_{n}\right\}$ and $\left\{\lambda_{n}\right\}$ such that $\lambda_{n} \uparrow \lambda_{0}$ and

$$
a_{n}=E_{x_{n}}\left[\exp \left(\lambda_{n} \sigma\right)\right] \rightarrow+\infty \quad \text { as } n \rightarrow \infty .
$$

Let $m_{n}$ be the Dirac measure $\delta\left(x_{n}\right)$, and put

$$
P_{n}=K_{\lambda_{n}} m_{n} / a_{n} \text {. }
$$

From (3.9) and (3.10), we have $P_{n} \in \Pi(\bar{S})$ and

$$
P_{n}=\lambda_{n} G^{*} P_{n}+m_{n} / a_{n} .
$$

Since $\Pi(\bar{S})$ is compact in the weak*-topology, we can take a subsequence of $\left\{P_{n}\right\}$ which converges to some element $P$ of $\Pi(\bar{S})$. From (3.11) and (3.12) $P$ must satisfy (3.8). By (2.5) $P$ is a probability measure on $S$. The theorem is proved.

Remark 3. For the existence of the above $P$, the condition (3.5) is not sufficient. To see this, consider the semigroup $e^{-k t} T_{t}$, where $\left(T_{t}\right)$ is a conservative semigroup. Then $\lambda_{0}=k$ and from (3.8) $P$ must be a finite invariant measure. However it does not exist in general. 
In the remainder of this paper, we always assume the conditions (A.1)-(A.3), and $P$ denotes the above probability measure.

THEOREM 3.7. We have

$$
\inf \{u / G u\} \leqq \lambda_{0} \leqq \sup \{u / G u\} \quad \text { for every } u \in C_{+}(\bar{S}) .
$$

Proof. Set $\lambda=\inf \{u / G u\}$. Since $u \geqq \lambda G u$, we have

$$
\lambda_{0} \int G u d P=\int u d P \geqq \lambda \int G u d P .
$$

By (2.6), we obtain $\lambda_{0} \geqq \lambda$. Similarly we can get the right hand inequality.

Remark 4. Since $A=-G^{-1}$, (3.13) is identical to (1.3). For the left hand inequality, we have

$$
\sup _{u \in C_{+}(\bar{S})} \inf (u / G u)=\lambda_{0} .
$$

To see this, let $u=E_{x}\left[e^{\lambda \sigma}\right]$ for $\lambda<\lambda_{0}$. Then we have $u=\lambda G u+1$, and so $u \geqq \lambda G u$, which proves (3.14).

Now we study the connection between $\lambda_{0}$ and the first eigenvalue.

Definition. A bounded continuous complex valued function $u$ is called an eigenfunction if it is nontrivial and satisfies

$$
u=\lambda G u,
$$

where $\lambda$ is some complex number which we call an eigenvalue.

THEOREM 3.8.

(i) If there exists a nonnegative eigenfunction, then the ergenvalue is $\lambda_{0}$.

(ii) Suppose that $\lambda_{0}$ is an eigenvalue. Then problem (3.15) has a unique normalized nonnegative eigenfunction. The eigenvalue $\lambda_{0}$ has the smallest real part of all eigenvalues and is simple.

Proof. (i) is clear from (3.13). Let $\lambda$ be a complex number and $T$; $=\int_{0}^{\infty} d t \exp (\lambda t) T_{t}$. By the definition of $\lambda_{0}, T_{\lambda}$ is bounded if $\operatorname{Re}(\lambda)<\lambda_{0}$. Therefore, if $\lambda$ is an eigenvalue then we have $\operatorname{Re}(\lambda) \geqq \lambda_{0}$. Let $u=\lambda_{0} G u$. Since $\lambda_{0}$ is real, we can assume that $u$ is a real function. Let $u^{+}=\max (u, 0)$. We can assume that $u^{+}$is nontrivial. Then we have

$$
\lambda_{0} G u^{+} \geqq \lambda_{0} G u=u \text {. }
$$

Thus we get $\lambda_{0} G u^{+} \geqq u^{+}$. On the other hand, by virtue of Theorem 3.6, we have 


$$
\int \lambda_{0} G u^{+} d P=\int u^{+} d P
$$

which implies $\lambda_{0} G u^{+}=u^{+}$by (2.6). From (2.6), $u^{+}$is positive on $S$ and so $u^{+}=u$. If $v$ is another eigenfunction, we set

$$
w=u \int v d P-v \int u d P .
$$

Then $w$ is also an eigenfunction and $w \geqq 0$ by the above argument and we have

$$
\int w d P=0
$$

which implies $w=0$. The uniqueness is proved.

Recall that if $\lambda_{0}$ is not simple, there exists a natural number $n \geqq 2$ such that

$$
\left(\lambda_{0} G-I\right)^{n} u=0 \text { and }\left(\lambda_{0} G-I\right)^{n-1} u \neq 0,
$$

where $I$ is the identity operator. Set $v=\left(\lambda_{0} G-I\right)^{n-1} u$ and $w=\left(\lambda_{0} G-I\right)^{n-2} u$. Then $v$ is an eigenfunction. On the other hand, we have

$$
\int v d P=\int \lambda_{0} G w d P-\int w d P=0,
$$

which is a contradiction. The theorem is proved.

Remark 5. The existence of the positive eigenfunction can be found in M. A. Krasnosel'skii [6] for the smooth diffusion process in a bounded domain with smooth boundary. The uniqueness and the simplicity of the first eigenfunction are also proved in it by a different manner.

\section{REFERENCES}

[1] BANDLE, M., Isoperimetric inequalities and applications, Boston-London-Melbourne, Pitman 1980.

[2] Donsker, M.D. ANd S.R.S. Varadhan, On the principal eigenvalue of secondorder elliptic differential operators, Comm. Pure Appl. Math. XXIX, 595-621 (1976).

[3] Friedman, A., Stochastic differential equations and applications, vol. 2, New York-London, Academic Press 1976.

[4] Grhman, I. I. ANd A. V. SKorohod, The theory of stochastic processes II, BerlinHeidelberg-New York, Springer 1975.

[5] KAC, M., On some connections between probability theory and differential and integral equations, Proc. 2nd Berkeley Symp. Math. Statist., Probability, 189-215 (1951).

-6] Krasnosel'skil, M. A., Positive solutions of operator equations, Groningen, Noordhoff 1964.

[7] Protter, M. H. and H.F. Weinberger, Maximum Principles in Differential Equations, Prentice-Hall, Englewood Cliffs, N. J., 1967. 
Department of Mathematics

FACULTY OF ENGINEERING

Tokyo Electrical Engineering College

Kanda-Nishikicho, Chiyoda-Ku, Tokyo 101

JAPAN 